\title{
Mast cell numbers in melanocytic naevi and cutaneous neurofibromas
}

\author{
N J Carr, A Y Warren
}

\begin{abstract}
This study aimed to determine the diagnostic utility of mast cell densities in distinguishing neurotised ("neural") melanocytic naevi from neurofibromas. Three groups of lesions were studied: neurofibromas, neural naevi, and naevi showing no neural change (control naevi). A Giemsa stain was used to demonstrate mast cells. The median mast cell density in the neurotised naevus group was significantly higher $(p<0.005)$ than that of both the neurofibroma and control naevus groups, but the distributions of the individual density counts overlapped considerably. The sensitivity and specificity of the mast cell density as a potential discriminator between neurotised naevi and neurofibromas, determined in relation to the optimal discrimination value obtained using Bayes' minimum cost decision rule, were low.

It is concluded that mast cell density on its own is of little use as a classification tool but could be of value in the context of a multivariate decision rule.
\end{abstract}

(F Clin Pathol 1993;46:86-87)

Neurotisation, or neural differentiation, is a well described feature of melanocytic naevi which occurs as the lesions mature. ${ }^{1-3}$ The naevus cells become spindled and show $\mathrm{S}$-shaped nuclei within eosinophilic cytoplasm; structures resembling nerve fascicles (known as neuroid tubes or lames foliacees) form, and a matrix of fibrillary collagen, together with a ground substance rich in mucopolysaccharides, develops. Dilated venules and numerous mast cells are also well described. A consequence of these changes is that neurotised naevi ("neural naevi") may simulate neurofibromas histologically.

If a melanocytic naevus consists entirely of neurotised areas the appearances may be indistinguishable from a neurofibroma in routinely stained sections. ${ }^{245} \mathrm{~A}$ definite distinction between them requires the use of specialised techniques, ${ }^{1256}$ such as immunohistochemical stains for myelin basic protein, which is found in neurofibromas but not melanocytic naevi, or electron microscopy to demonstrate melanosomes: neurotised melanocytes may retain their capacity to produce melanosomes, a feature not seen in neurofibromas. These techniques are relatively expensive and are not available in all diagnostic laboratories. This study was therefore designed to demonstrate whether a simple and readily available technique, namely a Giemsa stain to demonstrate mast cells, could be used to distinguish cutaneous neurofibromas from naevi showing complete neural differentiation.

\section{Methods}

All cases of cutaneous neurofibroma and melanocytic naevus submitted to the RAF Institute of Pathology and Tropical Medicine between January 1989 and March 1992 were retrieved from the files. They were examined microscopically to select suitable cases of neurofibromas, neurotised melanocytic naevi, and naevi showing no evidence of neurotisation (control naevi).

A total of 115 specimens were chosen from 103 patients, comprising 42 neurofibromas, 37 neural naevi, and 36 control naevi. The selection criteria were as follows. First, only lesions in which an area of at least $1.0 \mathrm{~mm}^{2}$ was suitable for study were included. Second, if the histological diagnosis was in doubt the case was excluded. Thus we only used cases in which the distinction between neurofibroma and neural naevus could be made on corroborative evidence, such as apparent origin from a nerve, the presence of residual typical naevus cells, or the presence of characteristic epidermal changes. Third, only neurotised naevi in which over half the lesion showed neural features were chosen. The control naevi were selected randomly from lesions showing no areas of neural change. No attempt was made to distinguish between congenital and acquired naevi; examples of neurotisation were seen in both types of lesion. The demographic data of the patients from which the lesions came are shown in table 1 .

The specimens had been fixed in $10 \%$ buffered formalin and processed to paraffin wax. All blocks were recut to provide sections at a uniform thickness of $5 \mu \mathrm{m}$. A Giemsa stain was performed in each case and the number of Giemsa positive mast cells $/ \mathrm{mm}^{2}$ was counted using an eyepiece graticule.

Only the neural parts of the partially neurotised naevi were counted. These parts tended to be the deeper areas. Therefore, counting of the control naevi was also concentrated in the deeper areas in an attempt to eliminate this as a possible confounding factor in the analysis.

\section{Results}

Mast cells were easily visualised by virtue of strong staining of their granules, and cell numbers could be counted without difficulty. The density of mast cells expressed as number of cells $/ \mathrm{mm}^{2}$ is shown for each of the three study groups in table 2 .

The Mann-Whitney $U$ test was used to 
Table 1 Patient details

\begin{tabular}{llll}
\hline & Neurofibromas & Neural naevi & Control naevi \\
\hline Total number of lesions & 42 & 37 & 36 \\
Total number of patients & 37 & 33 & 33 \\
Male:female ratio & $24: 13$ & $18: 15$ & $17: 16$ \\
Age range (years) & $21-64$ & $17-63$ & $10-62$ \\
Median age (years) & 38 & 35 & 27 \\
\hline
\end{tabular}

Table 2 Mast cell densities as number of mast cells counted $/ \mathrm{mm}^{2}$

\begin{tabular}{lccc}
\hline & Neurofibromas & Neural naevi & Control naevi \\
\hline Median mast cell & $47 \cdot 5$ & $108 \cdot 9$ & $57 \cdot 4$ \\
count (range) & $(5 \cdot 6-261 \cdot 7)$ & $(13 \cdot 3-413 \cdot 6)$ & $(11 \cdot 8-233 \cdot 3)$ \\
50 percentile range of counts & $23 \cdot 4-106 \cdot 8$ & $54 \cdot 4-171 \cdot 4$ & $32 \cdot 9-93 \cdot 0$ \\
\hline
\end{tabular}

assess the significance of differences between the three study groups in respect of the mast cell densities. There was a highly significant increase in mast cell densities in neural naevi compared with both neurofibromas ( $p=$ $0.0012)$ and control naevi $(p=0.0032)$. The difference between neurofibromas and control naevi was not significant ( $p=0.51$ ).

In an attempt to establish the usefulness of mast cell counts as a discriminator between neurofibromas and neural naevi, we determined the sensitivity and specificity in relation to an optimal discrimination value obtained using Bayes' minimum cost decision rule, assuming equal cost for misclassification and equal prevalences. ${ }^{7}$ The distribution of mast cell counts was significantly non-normal due to a right-tail skew in all three study groups, but a simple logarithmic transformation eliminated the distributional asymmetry that would otherwise have excluded the use of Bayes' rule. Analysis of the data from the neurofibromas and the neural naevi provided an optimal cutoff value of 58 mast cells $/ \mathrm{mm}^{2}$. Hence, if used in isolation, Bayes' rule suggests that lesions with mast cell densities below $58 / \mathrm{mm}^{2}$ should be classified as neurofibromas and lesions with densities above this value should be classified as neural naevi.

However, there was a considerable overlap in the mast cell density distributions making mast cell counts an inefficient way of classifying the lesions. This was reflected in the low sensitivity and specificity of the test: using 58 mast cells/ $\mathrm{mm}^{2}$ as the cutoff value, the observed rate of correct identification of neural naevi was $68 \%$ while the observed rate of correct identification of neurofibromas was $60 \%$.

\section{Discussion}

The distinction between a neurotised melanocytic naevus and a solitary neurofibroma may be of academic rather than practical interest in most instances. Nevertheless, we believe that accurate classification of lesions wherever possible is desirable on scientific grounds and, moreover, it may be of great importance in a case where the possibility of neurofibromatosis has been raised but not firmly diagnosed. The results of our study show that neural naevi have a significantly higher density of Giemsa positive mast cells than neurofibromas, but the range of mast cell numbers in each group is wide and there is a considerable overlap in the density distributions. A very high or very low mast cell density might be helpful in classifying a lesion, but we believe that, used in isolation, the technique is likely to be of only limited use in practice because of inadequate sensitivity and specificity.

The control naevi and neurofibromas in this study showed mast cell densities that were not significantly different from each other. These results are consistent with those of previous studies which have shown mast cells to be an important component of both neurofibromas $^{89}$ and melanocytic naevi. ${ }^{10}$ Our observations while counting mast cells suggested that their distribution within neurofibromas and naevi was not uniform. In both types of lesion mast cells tended to be found in highest concentration around blood vessels. In naevi they were also more likely to be found near adnexal structures. Some neurofibroma cases showed an increased concentration of mast cells towards the periphery of the lesion.

In naevi showing partial neurotisation it was the neural parts which showed the highest numbers of mast cells, apparently irrespective of the absolute mast cell density. These neural parts tended to be the deeper areas, reflecting the increased maturation of naevi with depth. However, many control naevi also exhibited higher mast cell counts with increasing depth, even though neural features were absent. We suggest that increasing maturity of naevus cells may be associated with increased numbers of mast cells and that this phenomenon may not necessarily depend on the development of a histologically neural phenotype.

In summary, neurotised naevi showed a significantly higher median mast cell count than either neurofibromas or non-neurotised naevi using the technique of counting Giemsa positive mast cell densities. However, the degree of overlap in the counts was so substantial that this finding is, in isolation, likely to be of little use in differential diagnosis in clinical practice. Nevertheless, mast cell density could provide useful diagnostic information if it were incorporated into a multivariate classification rule in association with other variables.

We thank the Director General of Medical services, Royal Air Force, for permission to publish this paper, Paul Strike for statistical help and advice, and the staff of the histopathology laboratory at the RAF Institute of Pathology and Tropical Medicine for their technical expertise

1 McKee PH. Melanocytic naevi and malignant melanoma In: McKee PH, ed. Pathology of the skin with clinical correlations. Philadelphia: JB Lippincott, 1989 13.1-13.36.

2 Lever WF, Schaumburg-Lever G. Histopathology of the skin. 7th edn. Philadelphia: JB Lippincott, 1989

Maize JC, Foster G. Age-related changes in melanocytic naevi. Clin Exp Dermatol 1979;4:49-58.

Becker SW. Diag Desis and treatment of pigmented nevi. Arch Dermatol Syph 1949;60:44-65.

5 Shelley WB, Arthur RP. Nerve fibres: a neglected component of intradermal cellular nevi. F Invest Dermatol ent of intradert

6 Penneys NS, Mogollon R, Kowalczyk A, Nadji M, Adachi K. A survey of cutaneous neural lesions for the presence of A survey of cutaneous neural lesions for the presence 7 Strike PW. Statistical methods in laboratory medicine. Oxford: Butterworth-Heinmann, 1991.

8 Johnson MD, Kamso-Pratt J, Federspiel CF, Whetsell WO. Mast cell and lymphoreticular infiltrates in neurofibromas. Comparison with nerve sheath tumours. Arch Pathol Lab Med 1989;113:1263-70.

9 Isaacson P. Mast cells in benign nerve sheath tumours f Pathol 1976;119:193-6.

10 Szekeres L. The possible role of mast cells in pigmented nevi. Arch Dermatol Forsch 1974;251:55-60. 